\title{
BIO-INSPIRED SEAMLESS VERTICAL HANDOVER ALGORITHM FOR VEHICULAR AD HOC NETWORKS
}

\author{
Mohanad M. Abdulwahhab ${ }^{1}$, Mohd Fadlee A. Rasid ${ }^{1,2}$ and Fazirulhisyam \\ Hashim $^{1,2}$ \\ ${ }^{1}$ Department of Computer and Communication Systems, Faculty of Engineering, \\ Universiti Putra Malaysia, Serdang, 43400, Selangor, Malaysia \\ ${ }^{2}$ Wireless and Photonics Networks (WiPNET) Research Centre, \\ Faculty of Engineering, Universiti Putra Malaysia, Serdang, 43400, Selangor, Malaysia
}

\begin{abstract}
One of the most important factors to implement VANET is by considering the variety of wireless networks available around the city as well as the vehicles traffic scenarios. However, by providing a diverse range of wireless access technologies, it is necessary to provide continuous network connectivity as well as selecting the most suitable network technology and performance. Many researchers have worked on building algorithms for selecting the best network to improve the handover process. However, with high-speed vehicles mobility, the vertical handover process became the most challenging task in order to achieve realtime network selection. This paper proposes a bio-inspired network selection algorithm influenced by insect's behaviour which combines Artificial Bee Colony $(A B C)$ and Particle Swarm Optimization (PSO). The proposed algorithm is applied to process multi-criteria parameters to evaluate the best available network and then execute the handover process seamlessly. The results demonstrate the benefits of the proposed Multi-Criteria ABC-PSO method by reducing the handover decision delays by $25 \%$. It gives the optimum performance in terms of network selections and reduces the handover latency by 14.5\%. The proposed algorithm also reduces the number of unnecessary handovers by $48 \%$ for three different mobility scenarios based on traffic environments (highway, urban and traffic jam).
\end{abstract}

\section{KEYWORDS}

Vehicular Ad Hoc Networks (VANETs), Vertical Handover, PSO, ABC, Seamless, Heterogeneous

\section{INTRODUCTION}

The next generation of wireless communication technologies, especially vehicular ad hoc networks are facing many challenges that need to be solved in order to be able to coexist with each other[1]. Seamless mobility is one of the challenges that needs to be addressed in order to provide the ability of uninterrupted inter-technology roaming for mobile nodes[2]. Mobile nodes roaming between different network technologies will lead to Vertical Handover (VHO) process that occurs within the link and network layers due to the demands of shifting the device interface as well as the network point of attachment. Several investigations and efforts in standardisation are being made by several institutions and individuals to design a final implementation of each of these communication layers[3]. However, for the specific purpose of VHO in a heterogeneous wireless environment, the information prepared for the link layer and network layer before any operations of handover will directly affect the significant handover performance and consequently lead to seamless handover. Another challenge is the network discovery time that can lead to a major delay in the handover process while scanning various radio channels on each interface and 
International Journal of Wireless \& Mobile Networks (IJWMN) Vol. 11, No. 5, October 2019

selecting the best among them. Hence, the concept of mobility in a heterogeneous wireless network implies the continuation of data transmission or maintaining an ongoing session while the point of attachment changes during VHO. The VHO happens when a Mobile Node (MN) roams between different networks and change the device interface (physical layer) which leads to different direction of data session in the user side as well as the network attachment point in the network side (network layer).

Our proposed schemes present a multi-criteria artificial bee colony hybrid with particle swarm optimisation algorithm for evaluating the information gathered from the mobile nodes in the handover. The algorithm will process and calculate the required handover timing for each node in advance, based on the mobile nodes' velocity and received signal strength to reduce the handover process latency. The information is used to support all vertical handover operations, which includes network discovery, handover decision, and handover implementation.

In the initial stage of the operation, the previous Access Point (AP) provides information about the neighbouring networks for the MN to quickly discover available networks. The second stage of the operation is selecting the best available network for the $\mathrm{MN}$ by considering its velocity and distance. The final operation of the proposed scheme involves the mobile node sharing the current information with its neighbouring nodes based on its current location. The schemes run on realtime simulation based on three mobility scenarios in city environment which are highway, urban and traffic jam scenario.

The remainder of this article is organised as follows; A brief overview of related handover algorithms in VANET together with related ABC and PSO are presented in the next section, followed by a section on details of the proposed scheme. The simulation setup and detailed analysis are discussed in the following section before the article is concluded in the last section.

\section{OVERVIEW ON HANDOVER IN VANET}

Handover occurs when a mobile node changes its session from one access point to another while its connection remains active. Several types of handovers can be performed in wireless networks. These comprise of vertical and horizontal, hard, soft and seamless, upward-vertical and downward vertical, network-controlled, mobile-controlled and mobile-assisted handovers.

When a mobile node is moving from WLAN to LTE, the handover occurred among different access-points of different networks. This is called vertical handover or inter-system handover. The vertical handover happens when the mobile node goes through heterogeneous cells of the wireless network[4]. Their heterogeneous cells have different specifications in terms of bandwidth, frequency of operation and data rate. Due to these differences, the vertical handover process becomes more challenging than a horizontal handover, especially in VANET. The vertical and horizontal handover operations are performed because the overlay network structure by each network expanded by increasing the size of the cells at higher levels within the hierarchy. Many works have categorized the handover management process into three stages[5]:

a) Network Discovery: the stage when the MN scans for the APs to collect the required data to initiate the handover process.

b) Handover Decision: the stage when the gathered data are used to determine and to select the best AP by considering several criteria while comparing with the predefined values. 
International Journal of Wireless \& Mobile Networks (IJWMN) Vol. 11, No. 5, October 2019

c) Handover Execution: after choosing the AP, this stage is to reserve the resources on the destination network and to complete the handover process.

The work presented here will focus on the multi-criteria context-aware handover decision strategy based on Received Signal Strength (RSS), velocity, available bandwidth and MN location.

\section{RELATED WORKS}

\subsection{Previous works on vertical handover in VANET}

The vertical handover decision algorithms can be categorised according to classifications and schemes [2, 6-7] as below and are summarised in Table (1):

a) RSS based Schemes.

b) QoS Schemes.

c) Intelligence-based Schemes.

d) Context-based Schemes.

Table 1. A comparative summary of vertical handover decision algorithms

\begin{tabular}{|l|l|l|l|}
\hline Categories & References & Advantages & Limitations \\
\hline $\begin{array}{l}\text { RSS based } \\
\text { Schemes }\end{array}$ & {$[8],[9],[10]$} & $\begin{array}{l}\text { Reduced handover failure, } \\
\text { unnecessary handover } \\
\text { avoidance }\end{array}$ & $\begin{array}{l}\text { Ping-Pong effect, high } \\
\text { link utilization, } \\
\text { increased handover } \\
\text { latency, } \\
\text { high packet loss }\end{array}$ \\
\hline $\begin{array}{l}\text { QoS based } \\
\text { Schemes }\end{array}$ & {$[2],[11]$} & $\begin{array}{l}\text { Higher throughput, } \\
\text { less packet loss }\end{array}$ & $\begin{array}{l}\text { High handover latency, } \\
\text { not applicable for high } \\
\text { speeds, ping-pong effect }\end{array}$ \\
\hline $\begin{array}{l}\text { Intelligence } \\
\text { based } \\
\text { Schemes }\end{array}$ & $\begin{array}{l}{[1],[12],[14],} \\
{[15],[16],} \\
{[17],[18]}\end{array}$ & $\begin{array}{l}\text { Reduced handover delay, } \\
\text { reduced packet loss, } \\
\text { intelligent network } \\
\text { selection }\end{array}$ & $\begin{array}{l}\text { Increased complexity, } \\
\text { higher decision } \\
\text { processing delays, } \\
\text { comparatively high } \\
\text { latency }\end{array}$ \\
\hline $\begin{array}{l}\text { Context- } \\
\text { based } \\
\text { Schemes }\end{array}$ & $\begin{array}{l}{[19],[20],} \\
{[21]}\end{array}$ & $\begin{array}{l}\text { Reduced packet loss, } \\
\text { optimal network selection, } \\
\text { embedded security, } \\
\text { reduced latency, } \\
\text { optimized throughput }\end{array}$ & $\begin{array}{l}\text { Supplementary } \\
\text { signalling, } \\
\text { context distribution, } \\
\text { higher resource } \\
\text { consumption }\end{array}$ \\
\hline
\end{tabular}

\section{a) RSS based Schemes:}

In 2017, a highway-based handover decision method was proposed to solve the unnecessary handovers and to reduce the vertical handover frequency by considering the nodes' velocity, jitter, position and density. In addition, it was also essential to evaluate the packet loss, network throughput and end-to-end delay to present a complete network performance [8]. 
International Journal of Wireless \& Mobile Networks (IJWMN) Vol. 11, No. 5, October 2019

In 2018, E.Smida et. al proposed a predictive SNR-based handover mechanism to solve the issue of handover latency by reducing the search for new Road Side Unit (RSU) time. In the handoff decision part, they considered the MN mobility tracking, neighbour RSUs information gathering, link quality and handover radius. However, the work did not addressed a major issue which is the ping pong effect of the SNR which leads to packet loss.

A study done in 2016 presented an Imperialist Competition Algorithm (ICA) of a vertical handover prediction method to reduce the unnecessary handovers. The ICA looked into the impact of RSSI on the number of handovers with the help of Ad Hoc On-Demand Distance Vector (AODV) protocol. However, this study did not provide an evaluation of handover performance in terms of latency and decision delay [10].

\section{b) QoS Schemes:}

Abdullah et al. proposed a multiple criteria vertical handover decision algorithm between three networks; LTE, WiMAX, and WLAN by using User Datagram Protocol (UDP). The results presented were based on reduction on the number of handovers and handover failure. However, the work did not consider the node speeds as well as handover latency nor apply this method on a variety of movement scenarios [2].

S. Chae et. al. proposed a handover scheme for high-speed mobility using coordinated multiple point transmission to provide seamless handover to improve Qos in VANET. However, the issue on ping pong effect when using the SNR in heterogeneous vehicular networks was not addressed [11].

\section{c) Intelligence-based Schemes:}

$\mathrm{Du}$ et al. proposed a vertical handover protocol between LTE and $802.11 \mathrm{p}$ networks by considering number of vehicles, end-to-end distance and fixed nodes' speed. Nevertheless, for a reliable handover performance, it is necessary to apply various vehicle speeds under several traffic movement scenarios [1].

F. Azzali et. al 2017 proposed a Fuzzy Logic algorithm for vertical handover decision in heterogeneous networks. The handover latency was measured in various velocities and had shown better results as compared to RSS based algorithm. However, the effects from the number of MNs and from the mobility scenario in the handover decision processing time need to be considered for more applicable algorithm [12].

In 2017, A. Gharsallah et. al. proposed a multi-criteria handoff algorithm for Vehicle to Vehicle (V2V) communications to enhance the AP selection in a heterogeneous network in order to reduce the handover failure. This study considered multiple vehicles speeds, RSS and traffic load to test the scheme [13].

Qamar et al. presented a Genetic Algorithm (GA) with Media Internet Protocol Version 6 (MIPV6) protocol to enhance the vertical handover process. The results were based on the service cost, Received Signal Strength Indicator (RSSI) and handover delay as compared to Ant Colony Optimisation (ACO) and Particle Swarm Optimisation (PSO). However, to evaluate network performance in terms of vertical handover, it is necessary to consider the effect of number and velocity of nodes and their impact on the handover process latency [14].

An Enhanced Advanced Duplicate Address Detection (EA-DAD) algorithm was proposed in 2015 by Abdullah et al. with the support of Fast Mobile Internet Protocol Version 6 (MIPV6) 
International Journal of Wireless \& Mobile Networks (IJWMN) Vol. 11, No. 5, October 2019

protocol to enhance the vertical handover process. The results show that the handover latency, MN speed, packet loss and signalling load were improved with the consideration of MN speed. However, the algorithm was only applied to a single mobile node [15].

In 2014, the Rapid VHO algorithm was proposed with the support of Proxy Mobile IPv6 (PMIPv6) protocol to improve the vertical handover process. This study evaluated the network performance by considering the effect of MN speed on handover latency and packet loss. Nevertheless, the impact of movement scenarios as well as the number of nodes were not considered in order to present a realistic performance [16].

Kim et al. presented a Fast Handover for PMIPv6 (PFMIPv6) protocol for vertical handover networks to enhance the reduction of packet loss, handover latency and signalling cost by considering the speed of nodes for performance evaluation in city and highway scenarios [17].

An Improved Genetic Algorithm-Simulated Annealing (IGA-SA) algorithm was proposed to solve the handover issues in heterogeneous network. The study revolved around Markov decision process applied to the handover decision stage to reduce the number of handovers. The results have shown the effect of RSS, the number of handovers and handover decision delay in a single scenario [18].

\section{d) Context-based Schemes:}

In terms of vertical handover in VANET, Goudarzi et al. proposed the Markov Decision ProcessBiogeography Based Optimisation (MDP-BBO) algorithm with Media-independent handover (MIH) protocol to enhance the vertical handover decision. This study covered several network parameters such as mobile nodes' velocities, handover signalling loads, packet loss, throughput, handover latency, signalling overhead, handover delays, computation time, handover failure and unnecessary handover rate. However, it did not consider the city environment that plays a significant role in the vehicular network performance in general, especially vertical handover [20]. Another work had concentrated on enhancing the vertical handover process by depending on a hybrid algorithm between BBO with the Markov chain and MIH (IEEE 802.21) protocol. The MDP-BBO algorithm was evaluated based on handover rate, handover failure, handover latency, packet loss and throughput against the vehicle speed and nodes density. Nevertheless, the simulation environment used was not very realistic since the vehicle's movement is set randomly without the consideration of road obstacles as well as movement scenarios [21].

The Neighbour Predictive Adaptive Handoff (NPAH) algorithm was proposed to ensure seamless node communication as well as providing a neighbours' predictive and adaptive handover. The NPAH discovers the weak links and establish handover process with the least resource-dependent node and considered the distance of neighbours. The results presented were based on the number of vehicles and speed such as packet loss, delivery factor and outage time [22].

Skondras et al. presented vertical handover management for cloud computing between LTE and $802.11 \mathrm{p}$. This study focused on deploying ten vehicles with a variety in velocity and bandwidth as well as considering the vehicle's services and operator's policies. The scheme aimed to evaluate the network based on packet loss, jitter, delay and throughput. However, while studying the vertical handover in cloud computing, it is necessary to have a variety in terms of the number of cars in order to evaluate the handover latency [23]. 


\subsection{Previous works on ABC in VANET}

Recently, by simulating the intelligent behaviour of the honey bee swarm, an Artificial Bee Colony (ABC) algorithm was proposed [24], [25]. The algorithm divides the artificial bees into three groups, which are the employed bees, the spectators and the explorers. The responsibility for flying and harvesting the food source that the swarm of bees is exploiting falls on the employed bees. The spectators wait in the hive and decide whether the food source is adequate or not. This is done by observing the dances performed by the employed bees. The explorers randomly search for a new source of nourishment through some internal motivations or possibly external cues.

In the $\mathrm{ABC}$ algorithm, the term food source represents the solution for each problem considered and represented by an n-dimensional real value vector in which the solution adjustment is related to the nectar quantity of the associated food resource. As with other intelligent swarm-based approaches, the $\mathrm{ABC}$ algorithm is a repetitive process. Initially, the approach generates random solutions or a population of food sources; then, it repeats the previous steps until the completion criteria are met [26].

In the phase of the bees being employed, the artificial bees look for a new source of food that has more nectar within the food source neighbourhoods in their memory. Whenever they find a nearby food source as defined in Equation (1), they would compare if the new nectar is superior to the previous one. The bee then stores the new position and forgets the previous one. Next, the regulation can be calculated as defined in Equation (2). After the new source of food is produced, its capacity is evaluated and a greedy selection is applied and compared with its matrix. After that, the employed bees share their information on the food source with the curious bees waiting in the hive by dancing in the dance area [27],[28]:

$$
V i j=x i j+9 i j \times(x i j-x k j)
$$

Where $k \in\{1,2 \ldots, \mathrm{BN}\}, \mathrm{BN}$ is the number of food sources that is equal to the number of bees used in each subgroup, ej $\in\{1,2 \ldots, \mathrm{D}\}$ is a randomly chosen index. While $k$ is randomly determined, it must be different from i. $\theta \mathrm{ij}$ is a random number between $-1,1$. It controls the production of a nearby food source around $x i j$, and the change represents the comparison of food positions visually close to the bee. Equation (1) shows that when the difference between parameters of $x i j$ and $x k j$ decreases, the disturbance at the $x i j$ position also decreases [27],[28]:

$$
\text { Fitness }(i)=\left\{\begin{array}{l}
\frac{1}{1+f(i)} \quad \text {,if } f(i) \geq 0 \\
1+a b s(f(i)), \text { otherwise }
\end{array}\right.
$$

In the onlooker bees' phase, depending on the information provided by the employed bees, the artificial bees choose their food sources as defined in equations ( 3 and 4$)$. In order to do this, a selection technique based on the physical state, such as the roulette wheel selection method can be used. After probabilistically choosing a food source for a spectator, a neighbourhood source is determined, and its appropriateness value is calculated. As in the phase of the employed bees, it applies a greedy selection between two sources [27],[28]: 
International Journal of Wireless \& Mobile Networks (IJWMN) Vol. 11, No. 5, October 2019

$$
\begin{gathered}
\operatorname{Prob}(i)=\frac{0.9 * \text { Fitness }(i)}{\max \text { (Fitness) }}+0.1 \\
\operatorname{Prob}(i)=\frac{\text { Fitness }(i)}{\sum_{n=1}^{S N} \text { Fitness }}
\end{gathered}
$$

In the phase of the scout bees, the employed bees whose solutions cannot be improved through a predetermined number of tests will change their function to become explorers and discard their solutions. The new explorers will start looking for other solutions, randomly using Equation (5). Sources that are initially poor or have become poor from exploitation are abandoned, and negative feedback behaviour emerges to balance positive feedback [27],[28]:

$$
x i j=x \operatorname{mind}+\operatorname{rand}(0,1) \times(x \max j-x \min j)
$$

The optimisation of the particle swarm of the artificial bee colony (ABC-PSO) presented in [19] provides a reasonable VHO with lower costs and delays, more available bandwidth and fewer number of handovers. The PSO algorithm could make a reasonably intelligent delivery decision based on the study of the network status. The target is to ensure the QoS of different VHO MNs for single and multiple attribute situations are on par. The algorithm aimed at minimising the total expected cost and minimising the average number of handovers by considering the speed of the $\mathrm{MN}$, the cost of access to the network and the switching cost of the vertical handover decision. However, the study did not focus on some significant factors in VANET for handover performance such as the number of nodes, traffic movement scenarios as well as the network throughput and packet loss.

\subsection{Previous works on PSO in VANET}

The particle swarm optimisation (PSO) is a simulation algorithm inspired by nature and influenced by the bird's behaviour that flying within a flock. The particles are represented by the individuals that traverse in the hyperdimensional search space. The behaviour of the swarming birds causes each particle to fly in the swarm relying on the information gathered by their experience. Each particle searches for a multidimensional search space in order to solve the problem and share its solution with the swarm. All the particles learn from their historical experience and information gathered. During the searching process, they fly into the parametric space of dimension $D$ of the problem. The particles in general, fly to better search region during the search process. This algorithm relies on the velocity vid and position xid to update the $d t h$ dimension of the ith particle. The below equations show the relationship between these criteria;

$$
\begin{aligned}
& \operatorname{vid}(t+1)=w \times \operatorname{vd}(t)+c 1 \times r 1 \times(\operatorname{pbid}(t)-x i d(t))+c 2 \times r 2 \times(\operatorname{gbd}(t)-\operatorname{xid}(t)) \\
& \operatorname{xid}(t+1)=\operatorname{xid}(t)+\operatorname{vid}(t)
\end{aligned}
$$

Where:

$\mathrm{c} 1$ and $\mathrm{c} 2$ are the acceleration constants

$\mathrm{r} 1$ and $\mathrm{r} 2$ are two uniformly distributed random numbers in 0,1 .

$\mathrm{xi}, \mathrm{xi} 1, \mathrm{xi} 2, x i d$ is the position of the ith particle.

vi, vi1, vi2, vid signifies the velocity of the ith particle.

pbesti, pbesti1, pbesti2, pbestid is the best previous position yielding the best fitness value for the ith particle. 
International Journal of Wireless \& Mobile Networks (IJWMN) Vol. 11, No. 5, October 2019

$\mathrm{Gb}, \mathrm{gb1}, \mathrm{gb} 2, \mathrm{gbD}$ is the best position discovered by the entire population.

$\mathrm{w}$ is the inertia weight used to balance between the global and local search capabilities.

The PSO algorithm has two main models, namely the local model and global model. The definition of the neighbourhood for each particle is different in these two models. The particles neighbourhood of the global model is composed of particles made by different particles. Van den Bergh and Engelbrecht [29] indicated that the speed of convergence in the global model is faster than the local model. However, the probability of getting stuck in local optima is greater than the local module. On the other hand, the vulnerability to the attraction of the excellent local is lower at the local level and its speed of convergence is slower compared to the global model.

An SPSO has been proposed as a Standard PSO module by Bratton [30] and Kennedy [31]. In SPSO, a local loop topology is used with many test problems and the simulation results revealed that the local model reliability is higher than the global model.

The update of PSO local model velocity is given by:

$$
\operatorname{vid}(\mathrm{t}+1)=\mathrm{w} \times \operatorname{vd}(\mathrm{t})+\mathrm{c} 1 \times \mathrm{r} 1 \times(\operatorname{pbid}(\mathrm{t})-\mathrm{xid}(\mathrm{t}))+\mathrm{c} 2 \times \mathrm{r} 2 \times(\operatorname{lbd}(\mathrm{t})-\mathrm{xid}(\mathrm{t}))
$$

The performance of the PSO is significantly influenced by the population topology. It determines how the particles share information or communicate with each other. Population topologies can be classified into static and dynamic topologies. For static topologies, communication structures were tested for random starts, wheels, rims, edges and reveals that the algorithm's performance varies in different problems depending on the topology used. The work by Kennedy [31] has tested many features of the social network topology in five test functions. After that, Mendes et al. presented a fully-informed FIPS PSO algorithm [32]. In FIPS, the particle uses the stochastic average of pbests of all its neighbours instead of using its lowest position.

Looking into how PSO was explored in VANET, the work by Hernafi et al. proposed and investigated a network selection algorithm inspired by PSO in terms of the geographic position of the vehicle and a shortest route selection method based on the ant colony [33]. The focus was on the vertical handover technique with the consideration of RSSI and the effect of the number of cars. However, it did not investigate the effect of the city environment and vehicle speed on the handover performance.

Goudarzi et al. presented a study in 2015 that uses the Curve Fitting based Particle Swarm Optimization (CF-PSO) algorithm for vertical handover decision making by considering the RSSI to enhance the throughput and to reduce the number of handovers [34].

A PSO algorithm modification was published by Ahuja et al. in 2015 to study the heterogeneous network handover by reflecting the RSSI impact on the network selecting time as well as the throughput and service cost. However, the speed of nodes and handover latency were not addressed [35].

The vertical handoff decision making has been investigated by Wang et al. and they proposed a Particle Swarm Optimization - Fuzzy Neural Network (PSO-FNN) algorithm. The algorithm considers the number of nodes, average load and the probability of handoff call blocking between UMTS and WLAN [36]. 
International Journal of Wireless \& Mobile Networks (IJWMN) Vol. 11, No. 5, October 2019

The multi-PSO algorithm with optimum mutation (MPSO-OM) was proposed by Wang et al. to improve a handover decision scheme [37]. The work had studied on the effect of node velocity with the link connectivity stability to achieve the best solution for handover. One of the missing performance evaluation in this work was on the effect of number of nodes to the handover performance.

\section{MethodS}

This article concentrates on enhancing the seamless vertical handover performance in terms of the decision, selection and implementation stages in order to minimise the delays while the MNs are roaming. A multi-criteria artificial bee colony with particle swarm optimisation algorithm has been proposed to contribute by reducing the latency that may occur during the handover stages based on the nodes' positions and velocities. In the initial stage of the algorithm, the network needs to start collecting information about the location of the vehicles and assume a random global and local best positions for the PSO equation. Then it divides the MNs into two groups as workers and onlookers, so that the $\mathrm{ABC}$ algorithm can start gathering the data required for PSO which are mainly the number of MNs, their speeds and the received signal strength. The data gathering is required in order to calculate the distances towards the next hop and choose the best currently available AP for each node. The second stage starts by scanning the nodes and comparing their positions with the PSO and choose the best AP based on the signal strength, bandwidth and velocity. The algorithm then share this information with all the nodes within the same swarm to calculate in advance where the handover will happen and to which AP. The third stage is when the handover process is required to initiate, at the instant the mobile node has the required information from the swarm to select the best network AP. The priority here is to the RSUs due to its higher data rate compared with LTE. However, when there is no best position AP nearby, the MN will maintain the current AP or change to LTE coverage depending on the environment. The last stage is when the criteria are met, the worker bees stop scouting and share the global best solution to the entire network. Finally, network discovery is enhanced due to the employed bees, the handover selection will be seamless due to the proactive information sharing, and the handover implementation become faster due to the advanced arrangement. The proposed stages of ABC PSO are explained as pseudocode in Table (2).

The aim of proposing the Multi Criteria ABC-PSO based vertical handover selection scheme is to focus on providing transparent mobility by supporting the seamless handover management for the MN. Many experiments have been conducted to investigate the performance of the proposed algorithm and compare them with the previous PSO schemes. Simulations were performed under different network environments and different parameters. The advantage of this study is that it is using multi-criteria to evaluate the available APs depending on the proactive information gathered about the mobile nodes and the available access points. These criteria are the nodes' speed, nodes' location, received signal strength and bandwidth. Based on the collected data, the best access point is selected and compared with the rest of the APs by the proposed algorithm. The algorithm has been simulated in real-time for three different movement scenarios to study its effect (highway, urban and traffic jam).

The proposed schemes measure the performance of each stage independently. The first stage is measured in terms of the relation between the number of handovers, vehicle velocity, number of mobile nodes and handover decision delay. The second stage is measurement in terms of the packet loss, packet delay and average throughput. The last stage is in terms of measuring the average handover latency. These parameters are selected because they can measure the extent of the improvement and impact that may influence the performance of the handover system. 
International Journal of Wireless \& Mobile Networks (IJWMN) Vol. 11, No. 5, October 2019

The proposed schemes are implemented using the OMNeT ++ 5.1.1, Veins 4.6, Sumo 0.30.0 with GSL 2.4 (GNU Scientific Library). The simulation area consists of 10 LTE towers and 20 RSUs distributed randomly to cover the whole area. 100 vehicles are roaming around at varying velocities based on the real-time city environment which could affect the speed of movement through, for example, traffic lights or parking lots. The RSUs are connected to the LTE towers via the core network. The MNs are equipped with two network interface cards, one for LTE and one for RSU.

Table 2. Pseudocode for vertical handover decision algorithm (ABC-PSO)

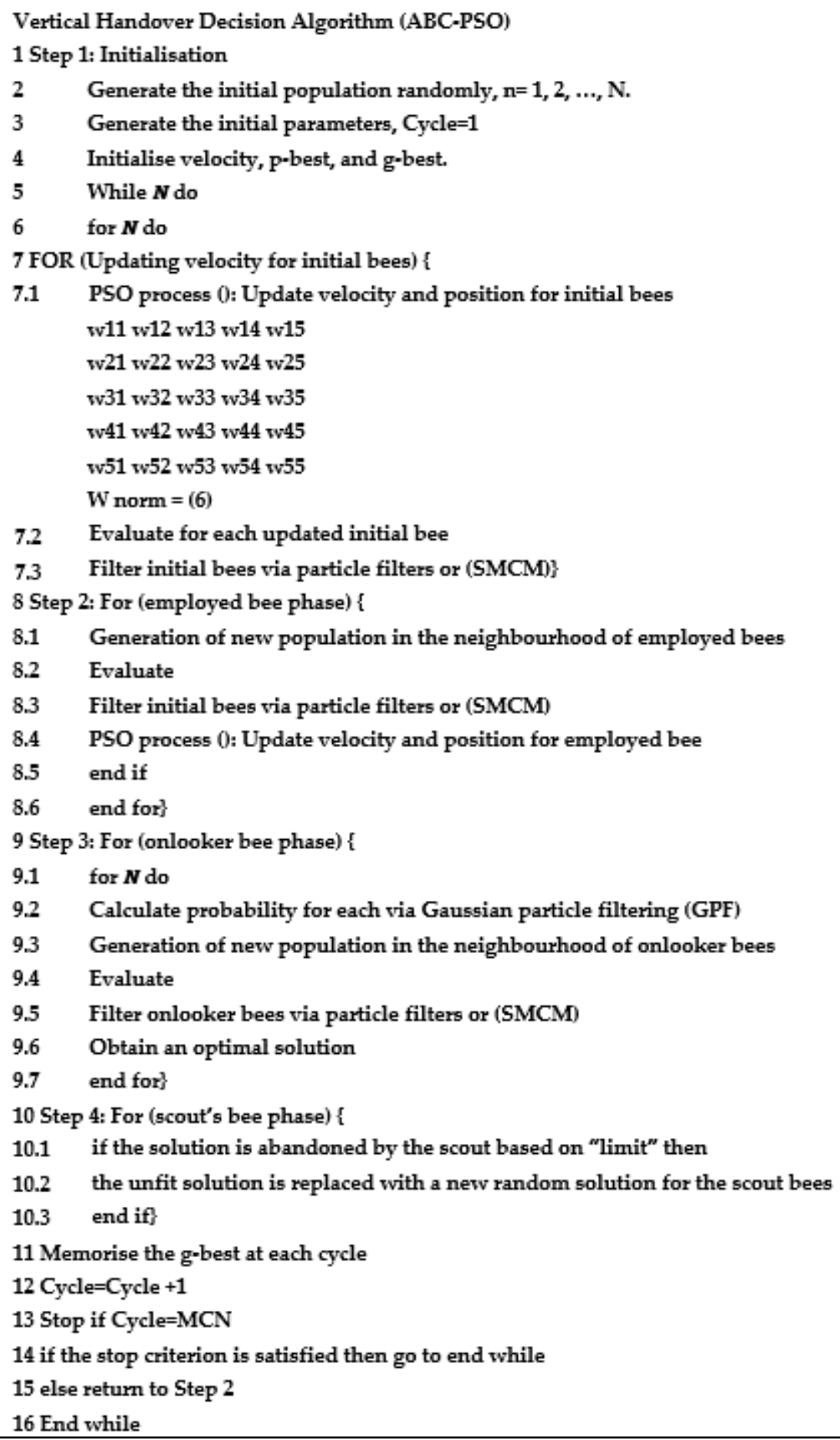


This simulation applies the IEEE $802.11 \mathrm{p}$ protocol for the data link layer (L2) because it is the protocol used in VANET. Table (3) shows the parameters used in this simulation. The mobile nodes are applied to the traffic scenarios simulated in SUMO to provide realistic movement. Furthermore, the mobility speed range is established on the highway between 60 and $120 \mathrm{~km}$ per hour, with the number of cars at 100. In urban cities, the car acceleration is $10 \mathrm{~km}$ per hour to a maximum speed of $60 \mathrm{~km}$ per hour, with the number of cars at 50 .

Meanwhile, in the traffic jam scenario, the minimum car speed is $0 \mathrm{~km}$ per hour, and the maximum speed is $30 \mathrm{~km}$ per hour, with the number of cars at 100 . One accident is set to occur every $30 \mathrm{sec}$ and lasts for $50 \mathrm{sec}$ in simulation time to ensure the traffic jam scenario. The summary of the simulation parameters is shown in Table (3) below.

Table 3. Simulation parameters

\begin{tabular}{|l|l|}
\hline Parameter Description & Parameter value \\
\hline Simulation area & $2500 \mathrm{~m} * 2500 \mathrm{~m} * 50 \mathrm{~m}$ \\
\hline Mobility model & Randomly restricted by roads map \\
\hline Pause time & 1 accident every $30 \mathrm{~s}$ and lasts for $50 \mathrm{~s}$ \\
\hline MN Speed & $\begin{array}{l}\text { Traffic jam } 0-30 \mathrm{~km} / \mathrm{h} \text {, Urban } 30-60 \mathrm{~km} / \mathrm{h}, \\
\text { Highway } 60-120 \mathrm{~km} / \mathrm{h}\end{array}$ \\
\hline MN Communication radius & $100 \mathrm{~m}$ \\
\hline $\begin{array}{l}\text { Number of RSUs / Cell coverage/ } \\
\text { bitrate }\end{array}$ & $20 / 150$ meters / 3-27 Mbps \\
\hline $\begin{array}{l}\text { Number of LTE towers /Cell } \\
\text { coverage / bitrate }\end{array}$ & $10 / 2000$ meters / 300 Mbps \\
\hline Number of cars & $5-100$ \\
\hline Data Packet size, control packet size & 6 Mbyte / 1080 byte \\
\hline Simulation time & 500 seconds \\
\hline
\end{tabular}

Some observation during the performance evaluation of the proposed algorithm is that the long execution and simulation time with the increase in the number of mobile nodes. The realistic traffic and movement environment provided by SUMO traffic simulator together with high exchange of data, formula calculations and criteria evaluation could have contributed to the long execution time when more mobile modes were simulated. These lead to the limitation during the evaluation of the proposed algorithm to a maximum of 100 mobile nodes in representing nodes density.

Depending on the requirements, the scenario contains four parts. First is the RSUs, the roadside units which represent the WLANs of the network. They are the small range high bandwidth units distributed randomly along the highways and the core of the city to provide the needed connection to the network that acts as the food source for the swarming nodes. They are responsible for collecting the information from the vehicles and share it with other nodes such as best positions and current locations that are needed for PSO. Secondly, the LTE towers that cover the whole simulation area provide the necessary connectivity when there is no RSU around the $\mathrm{MN}$ such as at inner roads or rural areas. It also collects the data in the environment and transmit it to the RSU and then to central networks. RSU and LTE towers are both connected to a network manager (routers) that runs the application such as traffic safety. Fourth is the world manager, 
International Journal of Wireless \& Mobile Networks (IJWMN) Vol. 11, No. 5, October 2019

which controls the imported data from SUMO to Veins such as the city map, building heights, and traffic lights. Part of the proposed work scenario in the simulation is visualised in Figure (1).

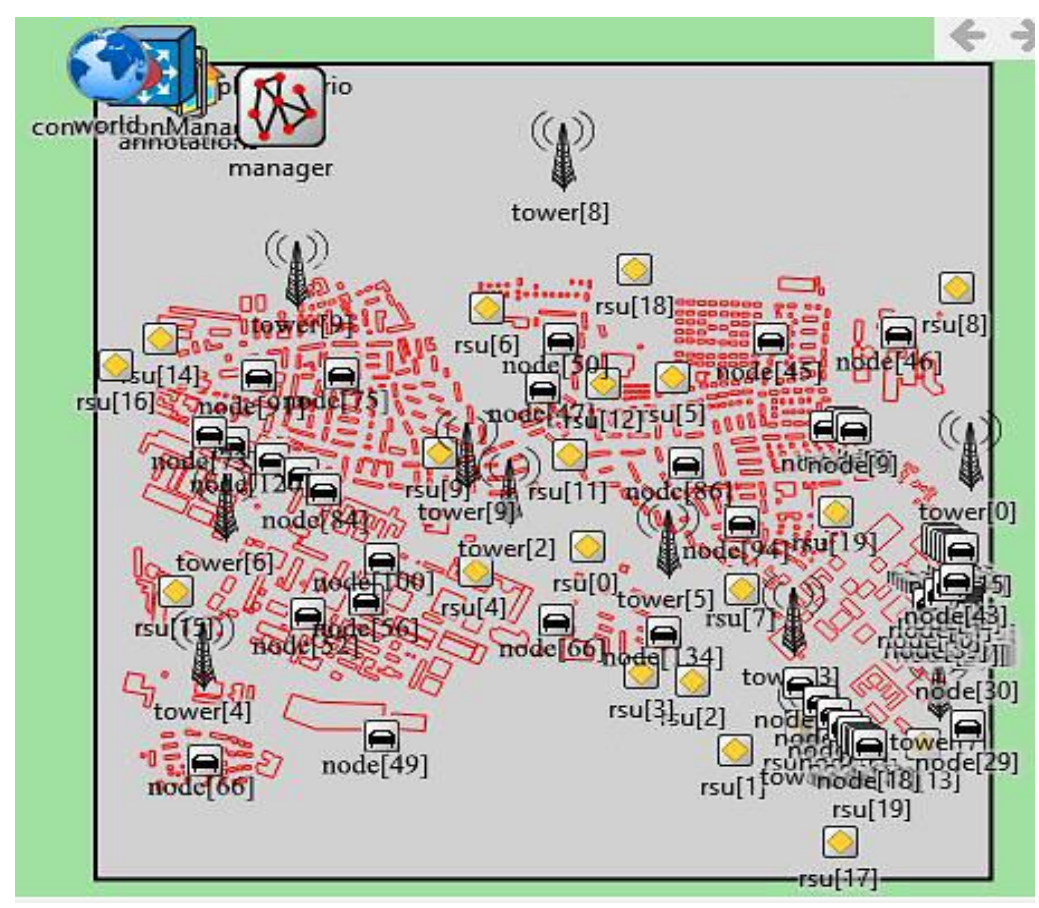

Figure 1. Part of the MC ABC-PSO scenario in OMNeT++

\section{RESULTS AND DISCUSSION}

In this section, different scenarios have been studied under different performance and city environment. With the benefits of applying traffic scenarios using the SUMO traffic simulator, three traffic conditions were used to study the effect on the proposed algorithm on network performance in terms of VANET. Figure (2) illustrates the effect of the three city environment on the handover latency with the increasing number of cars. Speed of cars is fixed to $100 \mathrm{~km} / \mathrm{h}$ for highway, fixed to $50 \mathrm{~km} / \mathrm{h}$ for urban and fixed to $20 \mathrm{~km} / \mathrm{h}$ for traffic jam.

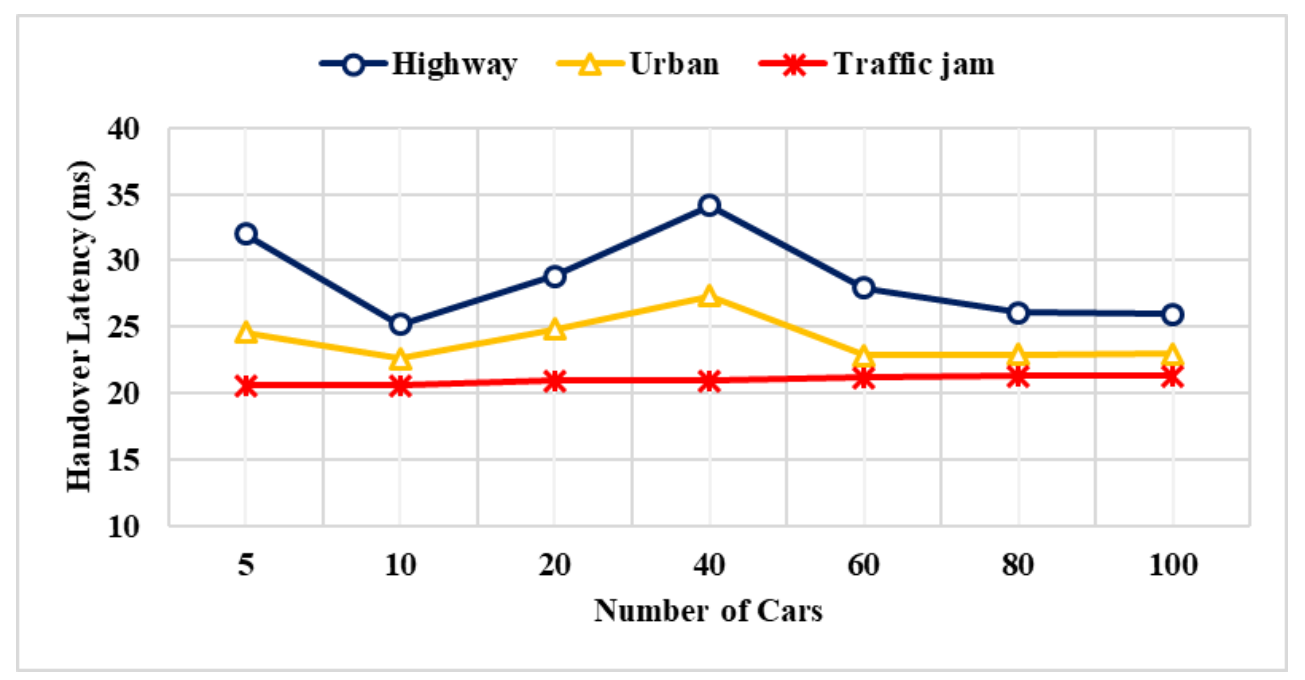

Figure 2. The effect of the number of cars on handover latency 
International Journal of Wireless \& Mobile Networks (IJWMN) Vol. 11, No. 5, October 2019

From Figure (2), the handover latency increases with the increase of car velocity due to the high demand of proactive data required to perform seamless handover and to prepare the MN to establish the next hop connection. Despite that, the number of cars was not a major concern in terms of handover latency. On the other hand, it can be noticed from the figure that the density of cars affects the handover latency and reached its peak at 40 cars before the latency started to decrease. This is due to the PSO algorithm, where the number of cars is one of the factors that affects performance. It is observed that the latency values are higher when there are fewer cars due to the rapid change in the best global position as the traffic is smooth and the bandwidth is freely available. Secondly, when the number of cars reach 40 while the ABC algorithm is dividing the cars into scouts and workers, the bandwidth usage and handover decision time will reduce and affect the latency. Moreover, after the increase in number of cars, it could be noticed on how the handover latency decreased due to the low demand on the bandwidth and the handover decision made smoothly since the global best position is calculated in advance with the assistance of car density. Do note that the higher the velocity, the faster the global best position will change with the car flow.

Figure (3) illustrates the relationship between the number of cars with the packet delivery delay time under different city scenarios, where the car speed is fixed to $100 \mathrm{~km} / \mathrm{h}$ in the highway, 50 $\mathrm{km} / \mathrm{h}$ in the urban setting, and $20 \mathrm{~km} / \mathrm{h}$ in the traffic jam.

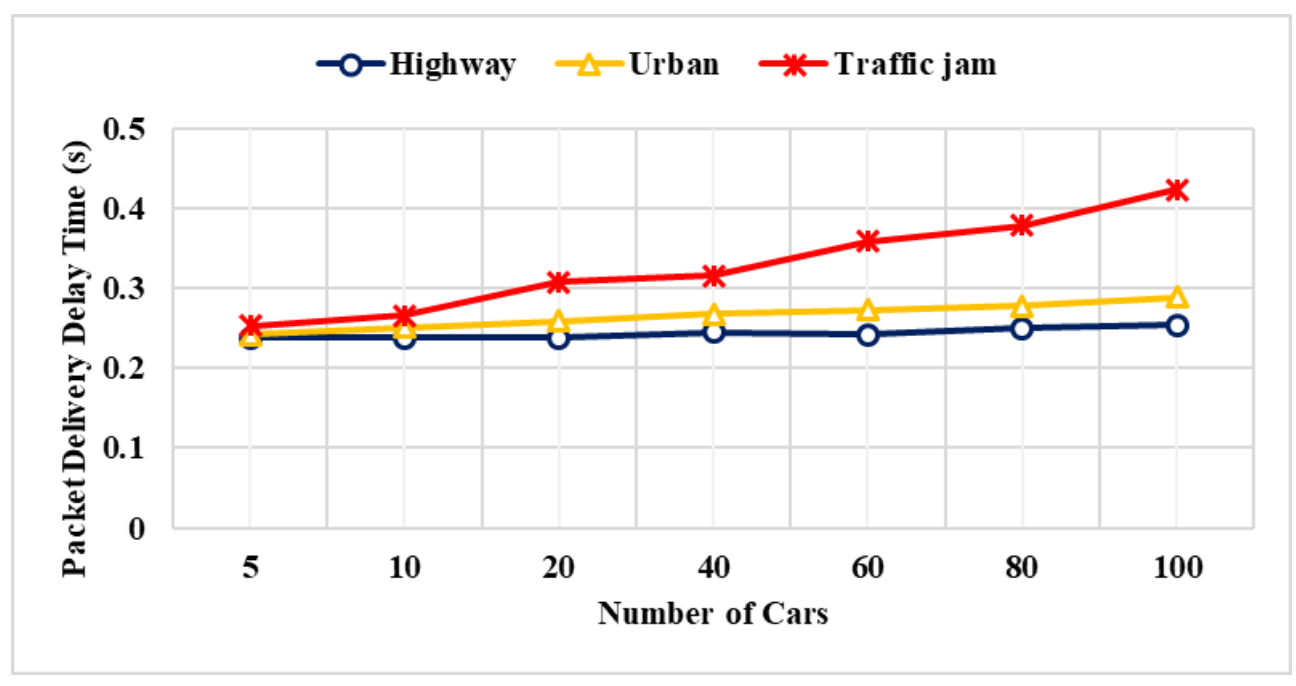

Figure 3. The effect of the number of cars on packet delivery delay time

From Figure (3), it can be seen how the number of cars impacts the packet delay time. With the increase of car density in a particular area, the packet delivery time increased as well due to the traffic load applied on specific APs. As for the highway scenario, it is obvious on how the smooth flow of cars gave less load to the APs which subsequently decrease the packet delivery time. The proposed algorithm is affected here with the increasing number of cars by the factor of bandwidth priority since it is one of the factors in selecting the best available AP to perform the handover. In the highway scenario, even when the density of cars is high, it can be noticed from the figure that the packet delivery time is still the same due to the best global position of PSO. This best global position has been chosen in advance with the help of scouting MNs of the ABC algorithm using the RSS and bandwidth factors. 


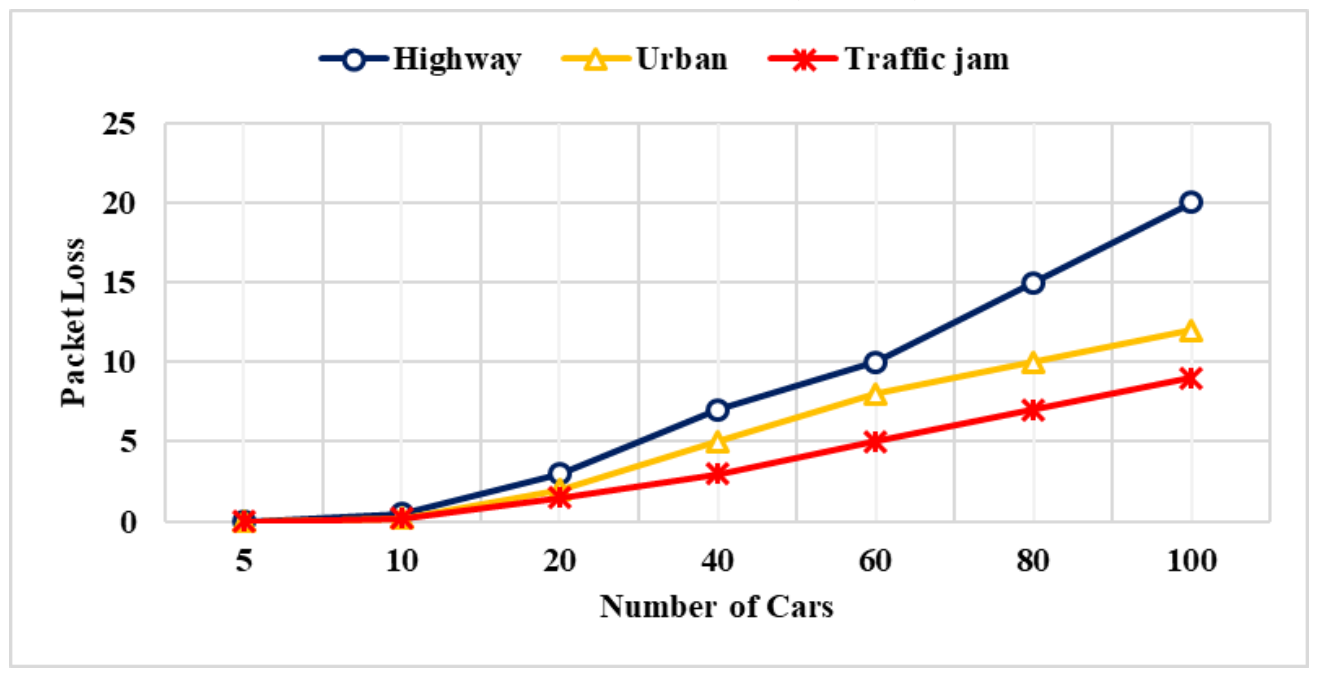

Figure 4. The packet loss vs the number of cars and velocity in three different scenarios

Figure (4) illustrates the packet loss for the MC ABC-PSO algorithm in VHO. Packet loss is defined as the amount of data packets that are sent from the source AP but failed to be received by the destination $\mathrm{MN}$ in a set duration of time. It can be noticed that with the increase of car speed, the packet loss ratio increase due to the high mobility and increase of handovers. The graph shows that the packet loss occurs while performing vertical handover. Since the vertical handover is happening between different networks (LTE and RSU), the previous AP sometimes will miss the MN disconnectivity when the MN moves away from the previous AP. The RSS decrease, thus the throughput of the connection is decreased causing more packet loss. The proposed algorithm found a way to reduce the packet loss by considering the RSS factor while selecting a global best solution.

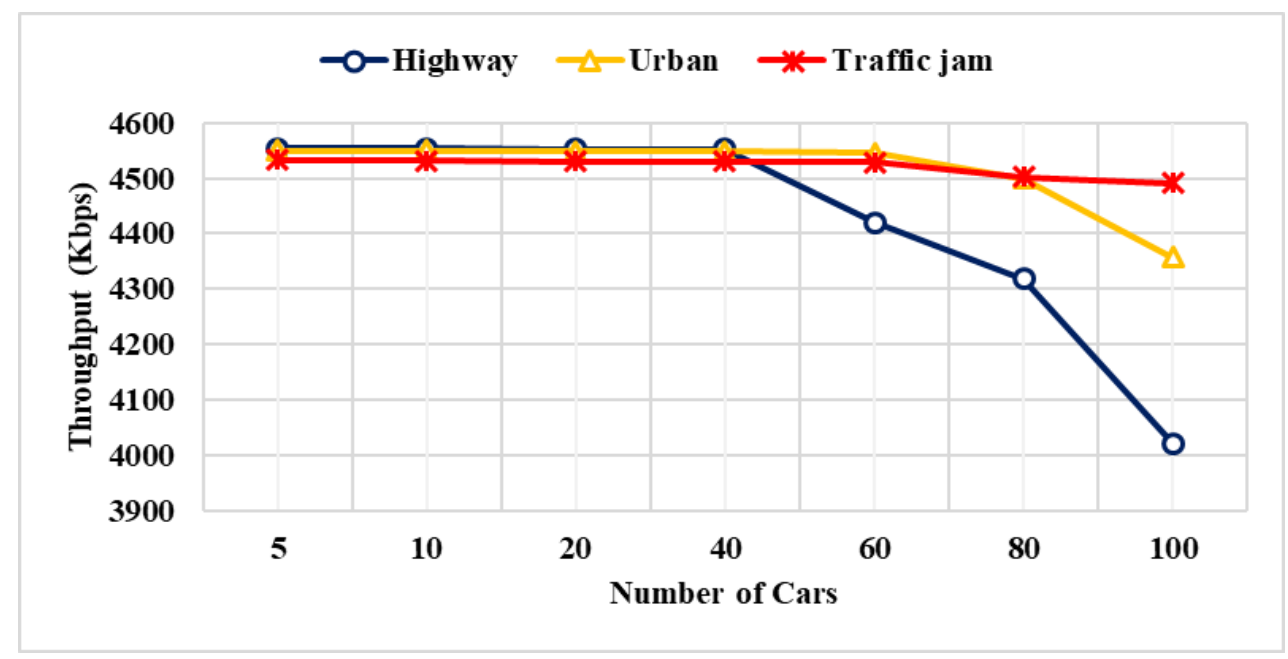

Figure 5. Comparison of the throughput with the number of cars in different scenarios

From Figure (5), it can be seen that the throughput decreases with the increase in car velocity, as observed in the highway scenario as compared to the urban and traffic jam. The number of cars starts affecting the throughput when it reached 50 cars which are considered acceptable due to the high flow of cars passing by the AP in urban city and highways. 


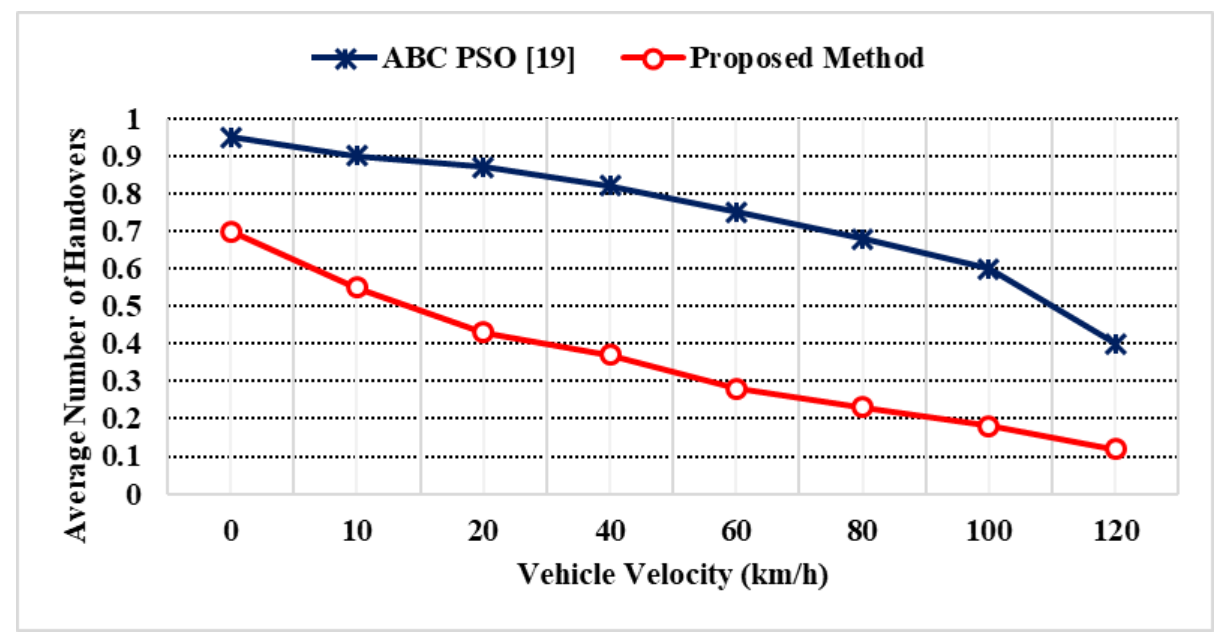

Figure 6. Average number of handovers vs vehicle velocity

From Figure (6), on average, the proposed MC ABC-PSO algorithm reduces the number of unnecessary handovers by $48 \%$. The reduction is achieved when reference [19] was used for comparison, using the same simulation parameters as used in [24]. This result can be clarified by the fact that the proposed algorithm considers the relationship between the MN velocity and the RSS as well as the network bandwidth when making decision to perform a handover. Since the ABC-PSO is based on finding the best solution from the gathered information, the RSS and velocity are the crucial factors here in evaluating the handover threshold. In order to reduce the unnecessary handovers, the proposed algorithm compares the predefined criteria with the updated information to eliminate the APs with low RSS, considers the pbest for other nodes and decreases the connection time with the current AP with the increase of average speed. When the average velocity decreases, the connection time extends, which causes a reduction in the handover possibility.

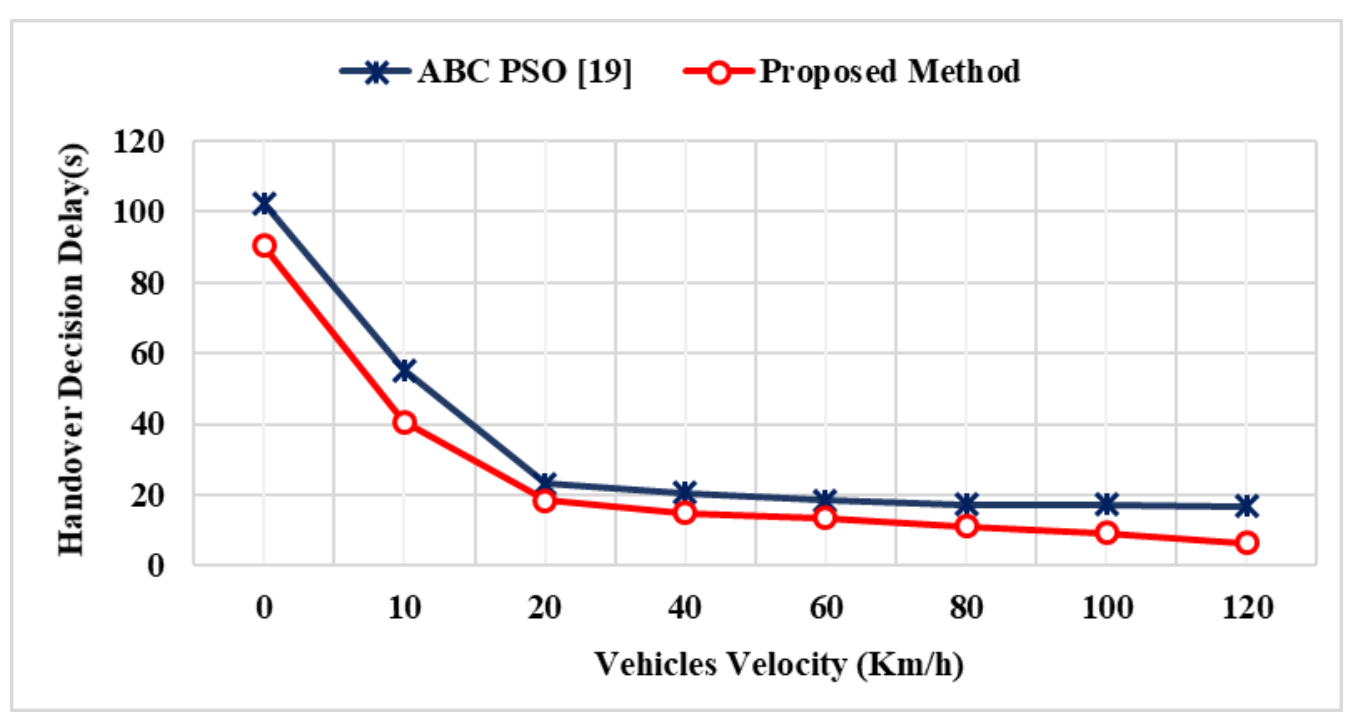

Figure 7. Average handover decision delay vs vehicles velocity 
International Journal of Wireless \& Mobile Networks (IJWMN) Vol. 11, No. 5, October 2019

From Figure (7), it can be observed that the proposed algorithm reduced the delay that occurs in handover decision by $25 \%$. The handover decision is made by considering three criteria in the proposed algorithm, which are the availability of access points RSS, the average velocity as well as the available bandwidth. By considering these criteria while finding the best solution in PSO as well as the cooperation among the mobile nodes in $\mathrm{ABC}$, the handover decision-making process could be minimized especially when the multi-criteria narrow the edges toward finding the best solution. When the global pbest is determined by the PSO, its information will be shared and considered by the MNs in order to perform its handover in advance, so that the MNs will save time in calculating the next hop when making its decision. Moreover, the impact of the handover threshold value, which varies based on $\mathrm{MN}$ speed, is increased.

\section{Conclusions}

As many of the related works were limited to a single traffic mobility scenario, this paper proposed a method that allow multiple mobility scenarios and consideration on scalability and dynamicity of mobile nodes. The work here proposed a bio-inspired handover algorithm focusing on the vertical handover process by providing a handover decision technique depending on the number of mobile nodes and velocity related to RSS, allowing seamless mobility in different movement scenarios based on the city environment (highway, urban and traffic jam). The proposed Multi Criteria ABC-PSO algorithm in this paper depends on predefining the best destination based on the current location as well as the swarm. The algorithm considers the MN velocity during the vertical handover decision process as well as the received signal strength. Besides, most of the current multi-criteria vertical handover algorithms were not able to predict MNs' circumstances dynamically. Furthermore, this approach managed to avoid unnecessary handovers by taking mobile nodes' velocities into account.

Extensive simulations were performed for different mobility scenarios based on traffic environments and the handover performance by using the Multi Criteria ABC-PSO approach was evaluated. By considering the nodes position and velocity, the remarkable findings from the simulation is that the proposed framework successfully minimized the vertical handover decision delay and latency, as well as the number of unnecessary handovers and packet loss rate. The most notable findings are the reduction in the number of unnecessary handovers by $48 \%$ as compared to the similar work and conventional approach.

Future work and improvement of the Multi Criteria ABC-PSO may consider clustering approach for the mobile nodes. The approach maybe able to reduce the calculation time by dividing the nodes into clusters that work as cloud computing. A central processing can then broadcast the concluded global best positions to the clusters based on their local best positions, ultimately towards improving the handover performance in vehicular networks.

\section{REFERENCES}

[1] W. Du and Q. Liu, 'Seamless Vertical Handoff Protocol for LTE- 802 . 11p Hybrid Vehicular Communications over the Tactile Internet', 2018 IEEE International Symposium on Haptic, Audio and Visual Environments and Games (HAVE), pp. 1-5, 2018.

[2] R. M. Abdullah and Z. A. Zukarnain, 'Enhanced handover decision algorithm in heterogeneous wireless network’, Sensors (Switzerland), vol. 17, no. 7, 2017.

[3] R. Duo, C. Wu, and T. Yoshinaga, 'SDN-based Handover Approach in IEEE 802 . 11p and LTE hybrid vehicular networks', 2018 IEEE SmartWorld, Ubiquitous Intelligence \& Computing, Advanced \& Trusted Computing, Scalable Computing \& Communications, Cloud \& Big Data 
International Journal of Wireless \& Mobile Networks (IJWMN) Vol. 11, No. 5, October 2019

Computing, Internet of People and Smart City Innovation (SmartWorld/SCALCOM/UIC/ATC/CBDCom/IOP/SCI), pp. 1870-1875, 2018.

[4] L. Nithyanandan and I. Parthiban, 'Vertical Handoff in WLAN-WIMAX-Lte Heterogeneous Networks Through Gateway Relocation', International Journal of Wireless \& Mobile Networks (IJWMN), vol. 4, no. 4, pp. 203-215, 2012.

[5] M. Mansouri and C. Leghris, 'Considering the Velocity in the Vertical Handover Network Selection Strategy', in Intelligent Technologies and Robotics, vol. 66, F. Khoukhi, M. Bahaj, and M. Ezziyyani, Eds. Cham: Springer International Publishing, 2019, pp. 37-42.

[6] F. Azzali, O. Ghazali, and M. H. Omar, 'Performance analysis of vertical handover in vehicular adhoc network using media independent handover services', Journal of Telecommunication, Electronic and Computer Engineering, vol. 9, no. 2-11, pp. 13-18, 2017.

[7] A. Ahmed, L. M. Boulahia, and D. Gaït, 'Enabling vertical handover decisions in heterogeneous wireless networks: A state-of-the-art and a classification', IEEE Communications Surveys and Tutorials, vol. 16, no. 2, pp. 776-811, 2014.

[8] M. Ali Hassoune, Z. Mekkakia Maaza, and S.-M. Senouci, 'Vertical Handover Decision Algorithm for Multimedia Streaming in VANET', Wireless Personal Communications, vol. 95, no. 4, pp. 42814299, 2017.

[9] E. B. Smida, S. G. Fantar, and H. Youssef, 'Predictive handoff mechanism for video streaming in a cloud-based urban vanet', Proceedings of IEEE/ACS International Conference on Computer Systems and Applications, AICCSA, vol. 2017-Octob, pp. 1170-1177, 2018.

[10] S. Goudarzi, W. H. Hassan, A. H. A. Hashim, S. A. Soleymani, M. H. Anisi, and O. M. Zakaria, 'A novel RSSI prediction using imperialist competition algorithm (ICA), radial basis function (RBF) and firefly algorithm (FFA) in wireless networks', PLoS ONE, vol. 11, no. 7, pp. 1-25, 2016.

[11] S. Chae, T. Nguyen, and Y. M. Jang, 'Seamless QoS-Enabled Handover Scheme Using CoMP in Fast Moving Vehicular Networks', International Journal of Distributed Sensor Networks, vol. 9, no. 12, pp. 1-8, Dec. 2013.

[12] F. Azzali, O. Ghazali, and M. H. Omar, 'Fuzzy Logic-based Intelligent Scheme for Enhancing QoS of Vertical Handover Decision in Vehicular Ad-hoc Networks', IOP Conference Series: Materials Science and Engineering, vol. 226, no. 1, p. 012081, Aug. 2017.

[13] A. Gharsallah, N. Omheni, K. Ghanmi, F. Zarai, and M. Neji, 'A Seamless Mobility Mechanism for V2V Communications', in 2017 IEEE/ACS 14th International Conference on Computer Systems and Applications (AICCSA), 2017, pp. 1063-1069.

[14] E. Qamar, F. Sajjad, S. Kouser, and S. Ahmed, 'an Optimized Handover Management System in 3G / 4G-Wlan Using Genetic Algorithm', International Journal on Information Technologies \& Security, vol. 9, no. 3, pp. 19-30, 2017.

[15] R. Abdullah, Z. Zukarnain, F. Masoumiyan, and A. Abdullah, 'Mobility and handover technique in heterogeneous wireless networks', Journal of Computer Science, vol. 11, no. 3, pp. 466-473, 2015.

[16] R. M. Abdullah, A. Abdullah, N. A. W. A. Hamid, M. Othman, and S. Subramaniam, 'The Rapid Vertical Handover for Efficient IPv6 Mobility Support in Heterogeneous Wireless Networks', Arabian Journal for Science and Engineering, vol. 39, no. 2, pp. 851-860, 2014.

[17] M.-S. Kim, S. Lee, D. Cypher, and N. Golmie, 'Performance analysis of fast handover for proxy Mobile IPv6', Information Sciences, vol. 219, pp. 208-224, Jan. 2013. 
International Journal of Wireless \& Mobile Networks (IJWMN) Vol. 11, No. 5, October 2019

[18] S. Goudarzi, W. H. Hassan, M. H. Anisi, and S. A. Soleymani, 'MDP-Based Network Selection Scheme by Genetic Algorithm and Simulated Annealing for Vertical-Handover in Heterogeneous Wireless Networks', Wireless Personal Communications, vol. 92, no. 2, pp. 399-436, 2017.

[19] S. Goudarzi et al., 'ABC-PSO for vertical handover in heterogeneous wireless networks', Neurocomputing, vol. 256, no. 2017, pp. 63-81, 2017.

[20] S. Goudarzi, M. H. Anisi, A. H. Abdullah, J. Lloret, S. A. Soleymani, and W. H. Hassan, 'A hybrid intelligent model for network selection in the industrial Internet of Things', Applied Soft Computing, vol. 74, pp. 529-546, Jan. 2019.

[21] S. Goudarzi, W. H. Hassan, M. H. Anisi, M. K. Khan, and S. A. Soleymani, 'Intelligent Technique for Seamless Vertical Handover in Vehicular Networks', Mobile Networks and Applications, vol. 23, no. 6, pp. 1462-1477, Dec. 2018.

[22] O. AlFarraj, A. Tolba, S. Alkhalaf, and A. AlZubi, 'Neighbor predictive adaptive handoff algorithm for improving mobility management in VANETs', Computer Networks, vol. 151, pp. 224-231, Mar. 2019.

[23] E. Skondras, A. Michalas, A. Sgora, and D. D. Vergados, 'A Vertical Handover management scheme for VANET Cloud Computing systems', Proceedings - IEEE Symposium on Computers and Communications, pp. 371-376, 2017.

[24] Dervis Karaboga, 'An Idea Based on Honey Bee Swarm for Numerical Optimization', Technical Report-TR06, vol. 200, pp. 1-10, 2005.

[25] D. Karaboga and C. Ozturk, 'A novel clustering approach: Artificial Bee Colony (ABC) algorithm', Applied Soft Computing Journal, vol. 11, no. 1, pp. 652-657, 2011.

[26] T. J. Hsieh, H. F. Hsiao, and W. C. Yeh, 'Forecasting stock markets using wavelet transforms and recurrent neural networks: An integrated system based on artificial bee colony algorithm', Applied Soft Computing Journal, vol. 11, no. 2, pp. 2510-2525, 2011.

[27] D. Wu and J. Zheng, 'A Dynamic Multistage Hybrid Swarm Intelligence Optimization Algorithm for Function Optimization', Discrete Dynamics in Nature and Society, vol. 2012, pp. 1-22, 2012.

[28] X. Shi, Y. Li, H. Li, R. Guan, L. Wang, and Y. Liang, 'An integrated algorithm based on artificial bee colony and particle swarm optimization', in 2010 Sixth International Conference on Natural Computation, 2010, no. 5, pp. 2586-2590.

[29] F. van den Bergh and A. P. Engelbrecht, 'A cooperative approach to participle swam optimization', IEEE Transactions on Evolutionary Computation, vol. 8, no. 3, pp. 225-239, 2004.

[30] D. Bratton and J. Kennedy, 'Defining a Standard for Particle Swarm Optimization', in 2007 IEEE Swarm Intelligence Symposium, 2007, pp. 120-127.

[31] J. Kennedy and R. Mendes, 'Population structure and particle swarm performance', in Proceedings of the 2002 Congress on Evolutionary Computation. CEC'02 (Cat. No.02TH8600), 2002, vol. 2, pp. 1671-1676.

[32] R. Mendes, J. Kennedy, and J. Neves, 'The fully informed particle swarm: Simpler, maybe better', IEEE Transactions on Evolutionary Computation, vol. 8, no. 3, pp. 204-210, 2004.

[33] Y. Hernafi, M. Ben Ahmed, and M. Bouhorma, 'ACO and PSO Algorithms for Developing a New Communication Model for VANET Applications in Smart Cities', Wireless Personal Communications, vol. 96, no. 2, pp. 2039-2075, Sep. 2017. 
International Journal of Wireless \& Mobile Networks (IJWMN) Vol. 11, No. 5, October 2019

[34] S. Goudarzi, W. H. Hassan, M. H. Anisi, S. A. Soleymani, and P. Shabanzadeh, 'A Novel Model on Curve Fitting and Particle Swarm Optimization for Vertical Handover in Heterogeneous Wireless Networks', Mathematical Problems in Engineering, vol. 2015, pp. 1-16, 2015.

[35] K. Ahuja, B. Singh, and R. Khanna, 'Particle swarm optimization based network selection in heterogeneous wireless environment', Wireless Personal Communications, vol. 83, no. 1, pp. 441$454,2015$.

[36] W. Nan, S. Wenxiao, F. Shaoshuai, and L. Shuxiang, 'PSO-FNN-Based Vertical Handoff Decision Algorithm in Heterogeneous Wireless Networks', Procedia Environmental Sciences, vol. 11, no. PART A, pp. 55-62, 2011.

[37] X. Wang, H. Zhao, M. Huang, Z. Wen, W. Wu, and X. Liu, 'ABC supported handover decision scheme based on multi-PSO with optimum mutation', Journal of Networks, vol. 6, no. 8, pp. 1153$1162,2011$.

\section{Authors}

MOHANAD M. ABDULWAHHAB received his BSc in computer engineering from Dijlah University, Baghdad, IQ. He is a master student at the Department of Computer and Communication Systems, Faculty of Engineering. His research interests are ad-hoc networks, vehicular networks, cellular communication.

MOHD FADLEE A. RASID received his $\mathrm{PhD}$ in electronic and electrical engineering (mobile communications) from Loughborough University, Leicestershire, UK. He is currently an associate professor at the Department of Computer and Communication Systems, Faculty of Engineering at Universiti Putra Malaysia. His research interests are mobile and vehicular communications, wireless medical sensors and low power wide area networks.

FAZIRULHISYAM HASHIM received the M.Sc. degree from Universiti Sains Malaysia, and the $\mathrm{PhD}$ degree in telecommunications engineering from the University of Sydney, Australia. He is currently an associate professor at the Universiti Putra Malaysia. His research primarily focuses on network security and QoS of heterogeneous mobile and cellular

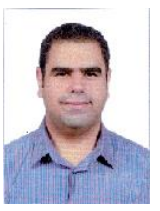
networks.
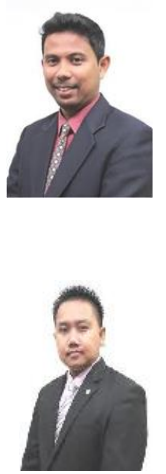